\title{
Frecuencia y coexistencia de patología rinosinusal en pacientes adultos roncadores
}

\author{
Frequence and coexistance of rhinosinusal pathology \\ in snoring adult patients
}

\author{
Claudia González $\mathrm{G}^{1}$, Ximena Fonseca $\mathrm{A}^{1}$, Constanza Beltrán $\mathrm{M}^{1}$, Marcela Arredondo $\mathrm{A}^{1}$, \\ Carla Napolitano $\mathrm{V}^{1}$.
}

\begin{abstract}
RESUMEN
Introducción: La etiología del ronquido es multifactorial. La vía aérea nasal está siendo cada vez más reconocida como un factor contribuyenteen alteraciones obstructivas del sueño.

Objetivo: Determinar la presencia de patología rinosinusal en roncadores, y determinar si hay diferencias entre pacientes con síndrome de apnea obstructiva del sueño (SAOS) y roncadores.

Material y método: Estudio retrospectivo y descriptivo de 220 pacientes.

Resultados: $B$ 50,5\% de pacientes presentaron patología rinosinusal relevante. Los diagnósticos más frecuentes fueron: rinitis alérgica 23,2\% , desviación septal 14,1\% y rinosinusitis $7,7 \%$. La rinitis alérgica fue más frecuente en pacientes roncadores. No se encontró otras diferencias entre ambos grupos.

Conclusión: Con excepción de rinitis alérgica, la patología rinosinusal no parece ser más frecuente en pacientes con SAOS que en pacientes roncadores.

Palabras clave: apnea del sueño, obstrucción nasal, roncopatía, nariz, rinosinusal.

ABSTRACT

Background: The etiology of snoring is multifactorial, although nasal airway is being more recognized as a contributing factor to sleep obstructive events.

Aim: To study the presence of rhinosinusal pathology in patients presenting with snoring, and to look for differences between patients with obstructive sleep apnea syndrome (OSAS) and with snoring.

Methods: Retrospective and descriptive study of 220 patients.

Results: $50.5 \%$ of the patients had relevant rhinosinusal pathology. The most frequent diagnoses were: Alergic rhinitis, 23,2\%; nasal septal deviation, 14,1\%, and rhinosinusitis, $7,7 \%$. Allergic rhinitis was more frequent in snoring patients. No other difference was found between both groups.

Conclusion: With the exception of allergic rhinitis, rhinosinusal pathology does not seem to be more frequent in patients with OSAS than in snoring patients.

Key words: Sleep apnea, nasal obstruction, snoring, nasal cavity, rhinosinusal.
\end{abstract}

'Médicos otorrinolaringólogos. Hospital Cínico Pontificia Universidad Católica de Chile. 


\section{INTRODUCCIÓN}

La consulta por ronquidos se ha incrementado dramáticamente durante los últimos años, debido al mejor conocimiento por parte del paciente de los riesgos asociados a la presencia del síndrome de apnea obstructiva del sueño (SAOS), tales como: hipertensión arterial, enfermedad cardiovascular, accidentes vasculares, somnolencia diurna, accidentes automovilísticos y disminución en la calidad de vida' ${ }^{1}$. La prevalencia del SAOS ha sido estimada en $2 \%-4 \%$ de los adultos en edad media?

En general se acepta que la etiología del ronquido es multifactorial, interviniendo factores como elasticidad de los tejidos, diferentes grados de obstrucción de vía aérea nasal, rinosinusal, orofaríngea y laríngea ${ }^{2}$. Es más frecuente en hombres $^{3}$, y en pacientes con antecedente de consumo de alcohol y medicamentos inductores del sueño.

Hay algunos métodos desarrollados con el objetivo de evaluar el grado de obstrucción anatómica determinado por las diferentes estructuras de la vía aérea en pacientes roncadores con sospecha o diagnóstico de apnea obstructiva del sueño tales como cefalometría frontal y lateral ${ }^{4}$ o evaluación volumétrica de vía aérea por tomografía computada 5 , entre otros. Pero la fre cuencia con que estos pacientes presentan enfermeda des rinosinusales que pueden favorecer la obstrucción de la vía aérea es desconocida

La vía aérea nasal está siendo cada vez más y más reconocida como un factor contribuyente en patología obstructiva del sueño. La resistencia de la vía aérea nasal es responsable del $60 \%$ o más de la resistencia total de la vía aérea, con la válvula nasal como el punto más importante en limitar el flujo aéreo ${ }^{6}$.

日 objetivo de nuestro estudio fue determinar y cuantificar la presencia de patología rinosinusal en roncadores, y determinar si hay diferencias entre pacientes con SAOS y roncadores.

\section{MATERIAL Y MÉTODO}

Se realizó un estudio descriptivo y retrospectivo. Se revisaron las fichas clínicas consecutivas de
220 pacientes que consultaron por ronquidos, con estudio polisomnográfico realizado en el Centro Médico del Sueño de la Pontificia Universidad Católica de Chile. Estos pacientes fueron evaluados por el mismo otorrinolaringólogo entre marzo 1998 y octubre 2002.

Se consignó edad, sexo, motivo de consulta al otorrinolaringólogo, historia rinosinusal previa, hallazgos al examen físico rinosinusal, índice de Masa Corporal (IMC), Índice de Apnea/Hipopnea (IAH), saturación de oxígeno mínima nocturna (SaOMN) y diagnóstico final.

En cuanto a los estudios complementarios estos se consignaron sólo cuando resultaron alterados. Se consideró que un paciente tenía una rinitis alérgica cuando el test cutáneo era positivo al menos para un alergeno (solicitado en aquellos pacientes que tenían síntomas sugerentes). Se estableció como desviación septal severa cuando la rinomanometría demostró obstrucción mecánica. $\mathrm{日}$ diagnóstico de sinusitis se realizó en base a los hallazgos de examen físico y anamnesis en los casos de sinusitis aguda y en la tomografía computada de cavidades paranasales en los casos de sinusitis crónica. Se compararon los hallazgos del examen rinosinusal y diagnósticos finales entre pacientes con SAOS y pacientes roncadores.

Síndrome de apnea obstructiva del sueño fue definido como la presencia de un Índice de Apneal Hipopnea $>5$ por hora en el polisomnograma ${ }^{7}$.

Los datos fueron almacenados en una base de datos Access 2000. Se realizó un análisis estadístico descriptivo y prueba de $\mathrm{Chi}^{2}$, considerando significativo un valor $p<0,05$.

\section{RESULTADOS}

\section{Caracterización del grupo estudiado}

日 grupo de estudio estuvo formado por 220 pacientes. De ellos, 29 (13,2\%) fueron mujeres y $191(86,8 \%)$ hombres. No hubo diferencia estadísticamente significativa al comparar pacientes con SAOS y roncadores en la distribución de hombres y mujeres ( $p=0,241)$. 
La edad promedio fue 46,6 $\pm 10,4$ años, con un rango de 14 a 70 años. En los pacientes con SAOS la edad promedio fue de $48 \pm 10,2$ años con un rango de 14 a 70 años y en los roncópatas la edad promedio fue de $41 \pm 10,4$ años con un rango de 24 a 60 años. Esta diferencia fue estadísticamente significativa con un valor $p<0,05$.

Las características generales de ambos grupos se resumen en Tabla 1. 1 hábito tabáquico no fue consignado en todas las fichas por lo que no fue posible considerarlo para el análisis.

De acuerdo al polisomnograma, el $100 \%$ de los pacientes eran roncadores, de ellos 43 (19,5\%) fueron roncadores sin apneas. Dos pacientes con síndrome de resistencia de la vía aérea superior fueron incluídos en este grupo. Los pacientes con SAOS fueron 177 (80,5\%). Las características del polisomnograma se resumen en Tabla 2 .

\section{Motivo de consulta y antecedentes rinosinusales}

日 principal motivo de consulta fue ronquidos con historia de apnea en 57,7\% ( $n=127)$ de los pacientes; ronquidos sin historia de apneas en $23,1 \%$ (n
$=51$ ); otras patologías otorrinolaringológicas en $16 \%$ ( $n=35$ ), en estos casos el diagnóstico de roncopatía fue sugerido por hallazgos del examen físico o por un motivo de consulta secundario del paciente o su acompañante; somnolencia diurna en $2,27 \%(n=5)$ y episodios de ahogo nocturno en $0,9 \%(n=2)$.

Del grupo total del estudio, 33,2\% tenían historia previa de patología rinosinusal (3 pacientes tenían rinitis y rinosinusitis $=1,4 \%$ ).

\section{Examen rinosinusal}

En el grupo total 108 (49\%) pacientes tenían examen físico rinosinusal normal y ausencia de síntomas rinosinusales y un paciente $(0,5 \%)$ tenía anosmia En consecuencia los 109 (49,5\%) pacientes antes mencionados fueron considerados con patología rinosinusal ausente o clínicamente irrelevante para roncopatía y los 111 pacientes restantes (50,5\%) presentaron patología rinosinusal relevante.

La frecuencia de anomalías en el examen rinosinusal fue: $22,3 \%$ hipertrofia de cornetes inferiores, $12,7 \%$ desviación septal obstructiva (demos-

Tabla 1. Características de la población en estudio

\begin{tabular}{|lccc|}
\hline & SAOS & Roncador & Valor $\mathrm{p}\left(\mathrm{Chi}^{2}\right)$ \\
\hline Hombres & $88,2 \%$ & $81,4 \%$ & 0,241 \\
Mujeres & $11,8 \%$ & $18,6 \%$ & 0,241 \\
IMC & $28,6 \pm 3,7$ & $27,9 \pm 4,6$ & 0,42 \\
\hline
\end{tabular}

SAOS: Síndrome de apnea obstructiva del sueño.

Tabla 2. Características polisomnográficas del grupo estudiado

\begin{tabular}{|lcllll|}
\hline & $\mathrm{N}(\%)$ & \multicolumn{1}{c}{ IAH } & \multicolumn{1}{c}{ Rango } & \multicolumn{1}{c|}{ SaOMN } & Rango \\
\hline RSA & $43(19,5 \%)$ & $1,98 \pm 1,8$ & $0-5$ & $87,8 \pm 4,9$ & $72-98$ \\
SAOS & $177(80,5 \%)$ & $39,2 \pm 24,9$ & $6-130,2$ & $77,9 \pm 9,5$ & $51-92$ \\
\hline
\end{tabular}

RSA: roncador sin apneas; SAOS: síndrome de apnea obstructiva del sueño; $\mathrm{N}$ : número de pacientes; \%: porcentaje; IAH: índice de apnea/hipopnea; SaOMN: saturación de oxígeno mínima nocturna. 
trada por rinomanometría) y 3,2\% poliposis nasal unilateral. La presencia de perforación septal fue significativamente mayor en pacientes roncadores. No hubo otras diferencias significativas entre roncadores y pacientes con SAOS (Tabla 3).

Considerando la historia, examen físico y pruebas complementarias, los siguientes diagnósticos fueron realizados para el grupo total del estudio: $23,2 \%$ rinitis alérgica; $12,7 \%$ desviación septal obstructiva; $7,7 \%$ rinosinusitis; $0,9 \%$ rinitis no alérgica; $0,5 \%$ epistaxis recurrente y $0,5 \%$ anosmia Sólo rinitis alérgica fue más frecuente en pacientes roncadores que pacientes con SAOS (Tabla 4).

En $76(34,5 \%)$ casos se observó coexistencia de más de una alteración.

\section{DISCUSIÓN}

Las características generales del grupo de pacientes roncadores fue muy similar a lo descrito en la literatura. La consulta por ronquido fue más frecuente en hombres, probablemente porque existe un subdiagnóstico en mujeres debido a la baja sospecha clínica y a que la población femenina consulta menos por roncopatía ${ }^{8}$.

Hasta ahora, se ha estudiado la presencia de factores anatómicos obstructivos que causan roncopatía, pero no hay publicaciones que evalúen la frecuencia de patología rinosinusal en roncadores. La obstrucción nasal ha sido asociada con roncopatía como un posible factor etiológico,

Tabla 3. Porcentaje total de alteraciones al examen físico

\begin{tabular}{|lcccl|}
\hline Diagnóstico & $\begin{array}{c}\text { Grupo total } \\
(\mathrm{n}=220)\end{array}$ & $\begin{array}{c}\mathrm{RSA} \\
(\mathrm{n}=43)\end{array}$ & $\begin{array}{c}\text { SAOS } \\
(\mathrm{n}=177)\end{array}$ & $\mathrm{P}$ \\
\hline Hipertrofia cornetes inferiores & $49(22,3 \%)$ & $11(25,6 \%)$ & $39(22 \%)$ & 0,61 \\
Desviación septal obstructiva & $28(12,7 \%)$ & $3(6,9 \%)$ & $25(14,1 \%)$ & 0,2 \\
Pólipos unilateral & $7(3,2 \%)$ & $2(4,7 \%)$ & $5(2,8 \%)$ & 0,5 \\
Poliposis nasal & $2(0,9 \%)$ & 0 & $2(1,1 \%)$ & 0,4 \\
Perforation septal & $3(1,4 \%)$ & $2(4,7 \%)$ & $1(0,6 \%)$ & $0,03^{*}$ \\
Tumor rinofaríngeo & $1(0,5 \%)$ & 0 & $1(0,6 \%)$ & 0,6 \\
Pinzamiento punta nasal & $1(0,5 \%)$ & 0 & $1(0,6 \%)$ & 0,6 \\
\hline
\end{tabular}

RSA: roncadores sin apneas; SAOS: síndrome de apnea obstructiva del sueño.

Tabla 4. Otros diagnósticos

\begin{tabular}{|lcccl|}
\hline Diagnóstico & $\begin{array}{c}\text { Grupo total } \\
(\mathrm{n}=220)\end{array}$ & $\begin{array}{c}\mathrm{RSA} \\
(\mathrm{n}=43)\end{array}$ & $\begin{array}{c}\text { SAOS } \\
(\mathrm{n}=177)\end{array}$ & $\mathrm{P}$ \\
\hline Rinitis alérgica & $51(23,2 \%)$ & $15(34,9 \%)$ & $36(20,3 \%)$ & $0,042^{*}$ \\
Rinosinusitis & $17(7,7 \%)$ & $6(13,9 \%)$ & $11(6,2 \%)$ & 0,08 \\
Rinitis vasomotora & $2(0,9 \%)$ & $1(2,3 \%)$ & $1(0,6 \%)$ & 0,27 \\
Epistaxis recurrente & $1(0,5 \%)$ & 0 & $1(0,6 \%)$ & 0,62 \\
Anosmia & $1(0,5 \%)$ & 0 & $1(0,6 \%)$ & 0,62 \\
\hline
\end{tabular}

RSA: roncadores sin apneas; SAOS: síndrome de apnea obstructiva del sueño. 
ya que cuando ésta es producida experimentalmente incrementa la resistencia al flujo aéreo y lleva a desórdenes del sueño y la respiración incluyendo apnea, hipopnea y ronquidos. Young y cols encontró que pacientes con obstrucción nasal debida a rinitis alérgica tienen 1,8 veces más probabilidad de presentar eventos severos de desórdenes del sueño y respiración que pacientes sin obstrucción nasal ${ }^{9}$.

Con estos antecedentes planteamos como hipótesis que los pacientes con SAOS podrían pre sentar mayor frecuencia o mayor número de patologías rinosinusales que los pacientes con roncopatía sin apneas, ya que esto podría permitirnos establecer una asociación causa-efecto.

Ambos grupos estudiados fueron comparables en cuanto a distribución por sexo e IMC. Sin embargo, los pacientes con SAOS son significativamente mayores en edad que los roncadores.

De todas las alteraciones y patologías rinosinusales objetivadas, sólo encontramos una mayor frecuencia de rinitis alérgica $(34,9 \%)$ y perforación septal $(4,7 \%)$ en roncadores comparado con pacientes con SAOS (20,3\%). Lo anterior sugiere cierta influencia de la rinitis alérgica en la producción del ronquido, pero no de las apneas, por lo que podríamos plantear que los elementos causantes del episodio de apnea no se encuentran en este nivel anatómico, sino que probablemente se encuentran localizados a nivel orofaringeo, laríngeo u otra área En consecuencia, no debemos esperar que el tratamiento aislado de una desviación septal, rinitis alérgica, rinosinusitis u otro, determine la desaparición de las apneas. Esto no significa que el hallazgo de patología nasal o sinusal no es importante en el tratamiento de este tipo de pacientes. Por el contrario, cualquier problema que cause algún grado de obstrucción nasal constituye otro factor más en la génesis del ronquido y debe ser correctamente diagnosticado y tratado. Estos hallazgos remarcan la importancia de una evaluación exhaustiva en busca de otras enfermedades, que por su naturaleza tienen un rol importante en obstrucción nasal y favorecen los ronquidos.

Considerando que el 50,5\% de los pacientes (roncadores y con SAOS) presentaban alteraciones rinosinusales relevantes, surge una nueva hipótesis: tal vez los pacientes con roncopatía y con SAOS tienen más alteraciones anatómicas nasales y mayor frecuencia de patología rinosinusal que los pacientes normales (sin roncopatía), lo que contribuye a la enfermedad. Esta pregunta no puede ser respondida por nuestro trabajo ya que carece de un grupo control, y por lo tanto deberá ser estudiada a futuro. Teniendo en cuenta esta limitación, compararamos la prevalencia reportada en la literatura para una patología rinosinusal específica en la población ge neral con las frecuencias observadas en nuestro estudio para los roncadores. La prevalencia reportada en la literatura de rinitis alérgica varía de un país a otro, en un rango de $14 \%{ }^{10}$ a $31 \%{ }^{11}$. En este estudio encontramos una frecuencia similar en pacientes roncadores $(23,2 \%)$. Estos hallazgos concuerdan con Kramer y cols, quién señaló que la rinitis alérgica no constituye un factor de riesgo mayor para un SAOS al no encontrar diferencia significativa en parámetros polisomnográficos entre pacientes alérgicos y no alérgicos ${ }^{12}$. La prevalencia de rinosinusitis o problemas sinusales reportada en 田U es $35 \%{ }^{13}$, un porcentaje mucho más alto que el 7,7\% observado en nuestro grupo. La prevalencia de poliposis nasal ha sido estimada en $1 \%$ a $2 \%$ en población adulta europea ${ }^{14}$, muy similar al 0,9\% encontrado en nuestros pacientes. Aparentementela rinitis alérgica, rinosinusits y poliposis nasal en roncadores y pacientes con SAOS tendrían una frecuencia similar a lo esperado para la población general. Será motivo de un estudio futuro determinar si estas alteraciones que generan obstrucción nasal son o no más frecuentes en pacientes con trastornos del sueño y la respiración que en la población general.

\section{CONCLUSIÓN}

Nuestros hallazgos sugieren que la patología rinosinusal, con excepción de la rinitis alérgica, parece tener similar frecuencia en pacientes roncadores que en pacientes con SAOS. Sin embargo se requieren estudios prospectivos para demostrar estos resultados. 


\section{Agradecimientos}

Al Dr. Jaime Godoy, del Centro Médico del Sueño de la Pontificia Universidad Católica de Chile, quién facilitó todas las copias de los polisomnogramas de los pacientes del estudio.

\section{BIBLIOGRAFÍA}

1. YOUNG T, PEPARD PE, GOTTLI円 DJ. Epidemiology of obstructive sleep apnea: a population health perspective. Am J Respir Crit Care Med 2002; 165: 1217-39.

2. StROLlo PJ, ROGers R. Ourrent concepts: obstructive sleep apnea. N Engl J Med 1996; 334: 99-104.

3. RELINE S, KUMP K, TISHLER PV, BROWNer I, FerRetTE V. Gender differences in sleep disordered breathing in a community-based sample. Am J Respir Crit Care Med 1994; 149: 722-6.

4. finkelsten Y, WeXler D, Horowitz E, Berger G NaChmani A, Shapiro-Fenberg M et al. Frontal and lateral cephalometry in patients with sleep-disordered breathing. Laryngoscope 2001; 111: 634-41.

5. CHen N, LI K, LI S, Wong CR, CHuAng ML, HWANG $\bigcirc$ ET AL. Airway assessment by volumetric computed tomography in snorers and subjects with obstructive sleep apnea in a fareast Asian population (Chinese). Laryngoscope 2002; 112: 721-6.
6. BUSABA N. The nose in snoring and obstructive sleep apnea Ourr Opin Otolaryngol Head Neck Surg 1999; 7: 11.

7. VAZ FRAGOSO C. Sleep disordered breathing. In: Conn's Qurrent Therapy, 54th ed, 2002; p.187.

8. WALKER RP, DURAZO-ARVIZU R, WAGTIIR B, GOPALSAMI C. Preoperative differences between male and female patients with sleep apnea. Laryngoscope 2001; 111: 1501-5.

9. Young T, FinN L, KIM H. Nasal obstruction as a risk factor for sleep-disordered breathing. $J$ Allergy Cin Immunol 1997; 99: 757-62.

10. ULRIK CS, VON LINSTOW ML, BACK $B$ V. Prevalence and predictors of rhinitis in Danish children and adolescents. Allergy 2000; 55: 1019-24.

11. ZHAO TB, WaNG HJ, OHeN Y, XIAO M, DUO L, LIU GET AL. Prevalence of childhood asthma, allergic rhinitis and eczema in Urumqi and Bejing. $J$ Paediatrics Child Health 2000; 36: 128-33.

12. Kramer MF, De La Chaux R, Dreier A, Pfrog N⿴R E, RASP G Allergic rhinitis does not constitute a risk factor for obstructive sleep apnea syndrome. Acta Aolaryngol 2001; 121: 494-9.

13. LIEU J, Fensten A. Confirmations and surprises in the association of tobacco use with sinusitis. Arch Atolaryngol Head Neck Surg 2000; 126: 940-6.

14. SÁnOHE-SEGURA A, BRIEVA J, RODRíGuE C. Regulation of immunoglobulin secretion by plasma cells infiltrating nasal polyps. Laryngoscope 2000; 110: 1183-8.

Dirección: Dra Gaudia González Gallardo

UDA Oorrinolaringología Pontificia Universidad Católica de Chile

Marcoleta $3522^{\circ}$ piso, Santiago, Chile

Tel: 56-2-6863484. Fax: 56-2-6399645

Email: clau_gonzalez@vtr.net 